\title{
Predicting recurrent ischemic mitral regurgitation: Through the 3-dimensional looking glass
}

\author{
David D. Yuh, MD, FACS, FACC
}

\author{
From the Section of Cardiac Surgery, Yale University School of Medicine, New Haven, Conn. \\ Disclosures: Author has nothing to disclose with regard to commercial support. \\ Received for publication June 27, 2016; accepted for publication June 27, 2016 \\ Address for reprints: David D. Yuh, MD, FACS, FACC, Section of Cardiac Surgery, Yale University School of \\ Medicine, 333 Cedar St, PO Box 208039, New Haven, CT 06520 (E-mail: david.yuh@yale.edu). \\ J Thorac Cardiovasc Surg 2016;152:860-1 \\ $0022-5223 / \$ 36.00$ \\ Copyright (c) 2016 by The American Association for Thoracic Surgery \\ http://dx.doi.org/10.1016/j.jtcvs.2016.06.049
}

The increasing use of 3-dimensional transesophageal echocardiography in evaluating structural heart disease has provided an opportunity to better understand complex pathologies, including ischemic mitral regurgitation (IMR). Wijdh-den Hamer and colleagues ${ }^{1}$ coupled 3-dimensional transesophageal echocardiography and advanced image modeling to identify the P3 tethering angle (P3TA) as a stronger independent predictor of IMR recurrence after mitral reduction annuloplasty than the posterior tethering angle measured by 2-dimensional echocardiography (2DE). The clinical relevance of accurately predicting IMR recurrence after repair has been heightened by recent evidence that demonstrates, among patients with severe IMR undergoing mitral surgery, no appreciable differences in left ventricular reverse remodeling or long-term survival between those treated with mitral valve replacement and those treated with repair. ${ }^{2,3}$

In addition to the analytic approach itself, an important contribution of the study by Wijdh-den Hamer and colleagues $^{1}$ is its demonstration of the influence of viewing plane selection in evaluating structural predictors of recurrent IMR and the notable limitations of preoperative 2DE in this regard. ${ }^{4-6}$ It illustrates a reasonable conceptual explanation behind the inability of preoperative 2DE to identify predictive parameters of leaflet tethering in the recent Cardiothoracic Surgical Network trial, ${ }^{7}$ which examined baseline clinical and echocardiographic predictors of recurrent IMR in a similar patient cohort. It is also quite interesting that different annuloplasty band conformations and characteristics did not seem to influence the rate of recurrence, reinforcing the importance of leaflet tethering in recurrence.

Further validation of this approach with larger patient cohorts and longer follow-up intervals will benefit from more quantitative assessments of IMR severity. Wijdh-den Hamer and colleagues ${ }^{1}$ rightly point out that using $2 \mathrm{DE}$ color flow Doppler jet area to grade recurrent IMR is subject to significant variability, stemming from a single apical 4-chamber view of a likely asymmetric regurgitant jet. More accurate assessments of recurrent IMR severity,

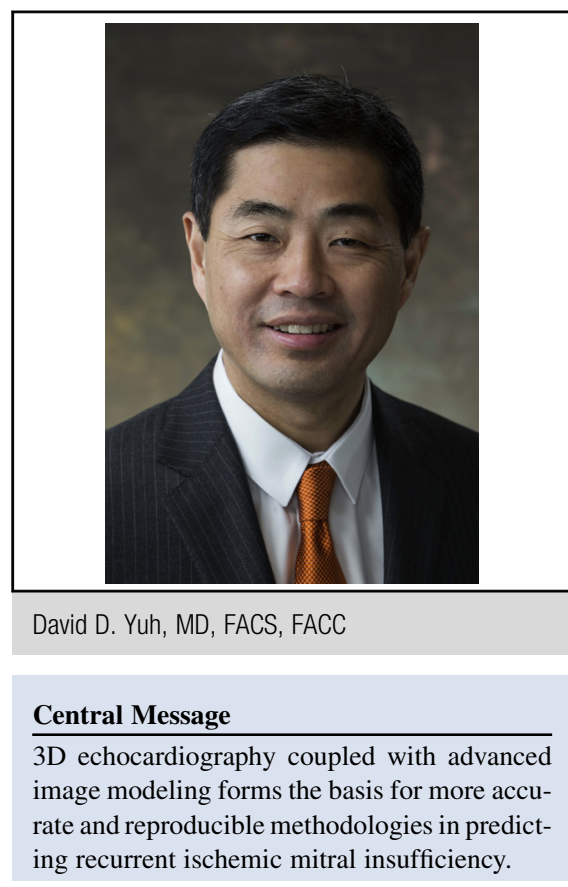

See Article page 847. possibly including cardiac magnetic resonance imaging quantification, may further increase the predictive resolution of this methodology. Furthermore, it would be very interesting to determine, in postrepair studies, whether the degree of change in P3TA resulting from the reduction annuloplasty is an independent predictor of IMR recurrence. This would further substantiate the influence of this geometric parameter on IMR recurrence and perhaps lead to more objective selection of annuloplasty band size and conformation to achieve a "nominal" P3TA.

The time-intensive, meticulous manual image segmentation required in the imaging and modeling approach used in this study are not readily translatable to routine clinical practice; however, it is virtually certain that automated segmentation algorithms will be built into future iterations of commercial echocardiographic analytic software. When this occurs, cardiac surgeons and their colleagues will be afforded a powerful quantitative tool for patient-specific preoperative surgical planning because other echocardiographic predictive parameters will likely be identified. Extension of this approach to catheter-based interventions for functional mitral regurgitation also seems likely. Taramasso and 
colleagues ${ }^{8}$ identified posterior leaflet restricted motion, as defined by a tethering angle $>45^{\circ}$, as an echocardiographic predictor of recurrent mitral insufficiency after MitraClip (Abbott Laboratories, Lake Bluff, Ill) application. Wijdh-den Hamer and colleagues ${ }^{1}$ demonstrates the exciting potential in fusing ever-improving imaging technology with sophisticated computational modeling.

\section{References}

1. Wijdh-den Hamer IJ, Bouma W, Lai EK, Levack MM, Shang EK, Pouch AM, et al. The value of preoperative 3-dimensional over 2-dimensional valve analysis in predicting recurrent ischemic mitral regurgitation after mitral annuloplasty. J Thorac Cardiovasc Surg. 2016;152:847-59.

2. Goldstein D, Moskowitz AJ, Gelijns AC, Aliawadi G, Parides MK, Perrault LP, et al. Two-year outcomes of surgical treatment of severe ischemic mitral regurgitation. $N$ Engl J Med. 2016;374:344-53.
3. Salmasi M, Acharya M, Humayun N, Baskaran D, Hubbard S, Vohra H Is valve repair preferable to valve replacement in ischaemic mitral regurgitation? A systematic review and meta analysis. Eur J Cardiothorac Surg. 2016;50: $17-28$.

4. Ereminiene E, Vaskelyte J, Benetis R, Stoskute N. Ischemic mitral valve repair: predictive significance of restrictive left ventricular diastolic filling. Echocardiography. 2005;22:217-24.

5. Matsunaga A, Tahta SA, Duran CM. Failure of reduction annuloplasty for functional ischemic mitral regurgitation. J Heart Valve Dis. 2004;13:390-7.

6. Zhu F, Otsuji Y, Yotsumoto G, Yuasa T, Ueno T, Yu B, et al. Mechanism of persistent ischemic mitral regurgitation after annuloplasty: importance of augmented posterior mitral leaflet tethering. Circulation. 2005;112:1396-401.

7. Kron I, Hung J, Overbey J, Bouchard D, Gelijns AC, Moskowitz AJ, et al Predicting recurrent mitral regurgitation after mitral valve repair for severe ischemic mitral regurgitation. J Thorac Cardiovasc Surg. 2015;149:752-61.e1.

8. Taramasso M, Denti P, Latib A, Guidotti A, Buzzati N, Pozzoli A, et al. Clinical and anatomical predictors of MitraClip therapy failure for functional mitral regurgitation: single central clip strategy in asmmetric tethering. Int $J$ Cardiol. 2015;186:286-8. 